\title{
Infrastructure as a Service (IaaS): A Comparative Performance Analysis of Open-Source Cloud Platforms
}

\author{
Sonia Shahzadi*, Muddesar Iqbal ${ }^{\dagger}$, Zia Ul Qayyum ${ }^{\ddagger}$, Tasos Dagiuklas ${ }^{\S}$ \\ *Email: s.shahzadi@swanmesh.com ${ }^{\dagger}$ Email: vc@uog.edu.pk $\ddagger_{\{}$Email: \{m.iqbal,tdagiuklas\}@1sbu.ac.uk \\ * Swan Mesh Networks Ltd \\ $\ddagger$ Pak-UK Institute of Emerging Technologies for Disaster Management \\ ${ }^{\dagger}$ School of Engineering, London South Bank University, UK
}

\begin{abstract}
Recently, cloud computing has been recognized as effective computing model, being adopted by both large corporations as well as SMEs (Small and Medium Enterprises). Due to its on-demand provisioning nature number of cloud based services are growing gradually and rapidly. The availability of open-source cloud computing platforms plays a vital role to enable researchers to go beyond state of the art for development of cloud applications. In this paper, we have provided an overview of cloud computing along with its services. We have presented a comparative performance analysis of different cloud IaaS platforms while covering different aspects of their application and service models. We have also proposed cloud test-bed using open-source cloud platform. A comparative performance analysis of open-source cloud platforms will assist researchers to select an appropriate tool to validate their research and experimentation.
\end{abstract}

Keywords-IaaS, PaaS, SaaS, CSP, NASA, OpenStack, DevStack, Oracle VM Virtual Box, Rally.

\section{INTRODUCTION}

Cloud computing is a dynamic field with a lot of flexibility and availability of very economical and reliable on-demand computing resources. The consumer can simply connect anytime from anywhere to access all the resources that are available through the cloud. These resources are managed and utilized in a distributed manner. Cloud computing is a model for enabling ubiquitous, convenient, on-demand network access to a shared pool of configurable computing resources (e.g. networks, servers, storage, applications, and services) that can be rapidly provisioned and released with minimal management effort or service provider interaction [1]. Essential characteristics of cloud computing are: on-demand self-service, broad network access, resource pooling, rapid elasticity and service measurement [1].

The main cloud computing deployment models are public, private, hybrid and community clouds [2]-[8]. By using these models, cloud computing provides different services such as Infrastructure as a Service (IaaS), Platform as a Service (PaaS) and Software as a Service (SaaS) [1]-[4], [7]-[11].

In IaaS, Cloud Service Providers (CSPs) offer the infrastructure as a service where a set of virtual computing resources, e.g. CPU, storage and network components are provided to the consumer [1]. Cloud users can deploy and run their own system and the software applications using these virtual resources [1] with access to the underlying hardware resources. The clients of IaaS have a reliable control on their infrastructures.
Examples of IaaS providers are Drop Box, Amazon EC2 and S3, Google Compute Engine, Rackspace, IBM Smartcloud, GoGrid and Akamai [2], [12], [13].

In PaaS, CSPs offer the platform as a service where customers can develop and deploy their own applications using development environment, application server technology, different supportive libraries and programming languages [1]. PaaS can be helpful where multiple clients use the same development environment. Integration with the databases and web services through some standards is also a feature of PaaS. The testing and the development can easily be automated for programmers in PaaS. The clients have full access and control over the tools and the developmental languages. PaaS provider's examples includes Windows Azure, Google App Engine, Red Hat OpenShift, Engine Yard, Heroku, AppFog, Caspio, MBaaS providers and Aptana Cloud [12].

SaaS is a demand based application that are hosted, maintained and managed by cloud service providers through a central location. Examples of SaaS providers are Zoho, Google Docs and Salesforce.com [9].

In the remainder of this paper, Section II present a comparisons of open-source cloud infrastructure platforms. In Section III, implementation of cloud testbed are described. Performance evaluation of two different OpenStack cloud implementations as a testbed are presented in Section IV. The paper is concluded in Section V.

\section{Literature ReVIEW}

\section{A. Cloud IaaS Frameworks}

Today, many cloud frameworks exist that are providing IaaS for both private and public clouds. Several, frameworks are available for this purpose including, Nimbus, Eucalyptus, OpenNebula and OpenStack. A taxonomy is proposed on selected cloud providers and survey is also given in [14]. From our literature analysis, we have identified some well-known cloud platforms that are providing IaaS. A brief overview of these platforms is given below:

1) Nimbus: Nimbus provides IaaS and it is divided into two phases that are: nimbus infrastructure and nimbus platform. Nimbus infrastructure is providing IaaS implementation that is compatible for EC2/S3. While Nimbus platform is providing additional tools for simplifying the infrastructure management. 
It is mostly considered for scientific cloud computing solutions [15].

2) Eucalyptus: Eucalyptus provides IaaS for private cloud. It has following five components [16], [6], [17]:

- Cloud Controller (CLC): It manages the virtual resources.

- Cluster Controller (CC): It controls the execution of VMs.

- $\quad$ Storage Controller (SC): It provides storage system which is called walrus.

- $\quad$ Elastic Block Storage (EBS): It provides Amazon EBS semantics support.

- Node Controller (NC): It controls VM activities including VM termination and VM execution.

3) OpenNebula: OpenNebula is an open source IaaS toolkit [18]. It can deal dynamic resource needs, snapshotting and live migration [6]. It supports different access interfaces, including OCCI service interface, REST-based interfaces and emerging cloud API standards.

4) OpenStack: OpenStack provides IaaS for private and public clouds. OpneStack is a combination of multiple opensource projects to develop your own cloud infrastructure and it is initiated by Rackspace and NASA in 2010 [19]. OpenStack main characteristics are: scalable, compatible and flexible, and open source [13]. It exploits various open-source projects and manages both compute and storage resources in the cloud [20]. OpenStack has mainly following components: Keystone (Authentication Service), Glance (Image Service), Nova (Compute Service), Neutron (Network Service) Horizon (Dashboard Service), Cinder (Block Storage) and Swift (Object Storage) [21], [16], [22], [23].

5) CloudStack: CloudStack originally developed by cloud.com in 2010 under GPL v3 license. In 2011, Citrix purchased Cloud.com and later on, in 2012, its license changed into Apache 2.0 when Citrix donated CloudStack to the Apache Software Foundation [16]. It consists of the following components [3], [16].

- Pods: Hardware configuration into a form of clusters and a pod can be consist of one or more clusters.

- Clusters: A group of multiple identical hosts and specifies for primary storage.

- Primary Storage: Used in each cluster for host VM instances.

- $\quad$ Secondary Storage: Used for VM images and snapshots storage.

- Availability Zones: Used to represent geographical locations which are helpful for VM allocation.

6) AbiCloud: AbiCloud provides IaaS and mainly it consists of following three components [3], [4]:

- AbiCloud Server: Used for computer management.

- $\quad$ AbiCloud Web Services (WS): Used to manage virtual applications.
- $\quad$ Virtual System Monitor (VWS):Used to monitor virtual infrastructure.

7) XEN Cloud Platform (XCP): $\mathrm{XCP}$ is an open-source solution for server virtualization and cloud computing platform includes the XEN hypervisor [24]. It doesn't provide a complete cloud infrastructure solution, but it is helpful for cloud management system [25].

\section{B. Comparison of IaaS Frameworks}

In order to get comparison of these platforms, we have derived certain assessment criteria from different cloud features e.g. structure, supported cloud models and programming languages. To perform networking tasks in cloud computing is also a significant part so it's essential to understand which network service is offered in each platform. Another important aspect is to check these platform compatibility with other hypervisors, and CSP's as well as scalability, load balancing and fault-tolerance. In Table I, we have done some features comparison that is derived from literature analysis [3], [4], [6], [15]-[18], [24]-[36]. The comparison provides the knowledge and understanding which makes it much easier for users, developers and the administrator's to choose the best suited framework that is compatible for their environment. Therefore, the presented review covers different characteristics and implementation aspects to help in developing better understanding of these platforms.

\section{IMPLEMENTATION OF ClOUd TESTBED}

In this section we have selected two of the OpenStack implementations i.e. deployment using DevStack and native 3-tier OpenStack deployment. Many researchers use DevStack to quickly create an OpenStack development environment for experimentation, whereas 3-tier OpenStack implementations are used for production of IaaS platform to implement as a research testbed to perform a performance comparison analysis. Using DevStack to substitute the OpenStack implementation can produce different results using the deployment environment. This comparative performance analysis will enable researchers to understand this difference among both the options available for testbed deployment in terms of their performance in lab environment for experimentation. Following tools are used to develop these testbeds.

- Cloud IaaS Platform: OpenStack and DevStack.

- Host OS: Ubuntu Server 14.04.

- Hypervisor: Oracle VM Virtual Box.

\section{PERFormance EVAluation}

We have used Rally benchmark tool to measure the performance of different cloud services to test and verify the scalability of cloud. Although, DevStack is not applicable for production environment, but if a user want to deploy a mini private cloud to seek certain cloud performance tests, then this work is beneficial for the purpose where user can get an indication on how well these cloud platform services can perform in different environments and use-cases. Furthermore, it will help the researchers to choose the right platform to setup their testbed in order to validate their hypothesis. We have performed test using same scenarios configuration for both testbeds to generate the results. 
Scenario 1: In scenario 1, we have run 100 iterations with 8 atomic actions to measure certain keystone services correctness and performance. As, shown in Figure 1, x-axis represents number of iterations while the $y$-axis represents the total duration times to complete the specific iteration in seconds and maximum time is 64.95 ( $\mathrm{sec}$ ) in DevStack case while 19.21 (sec) in OpenStack. As, this show that OpenStack performance is much better than as compare to DevStack in terms of keystone services.

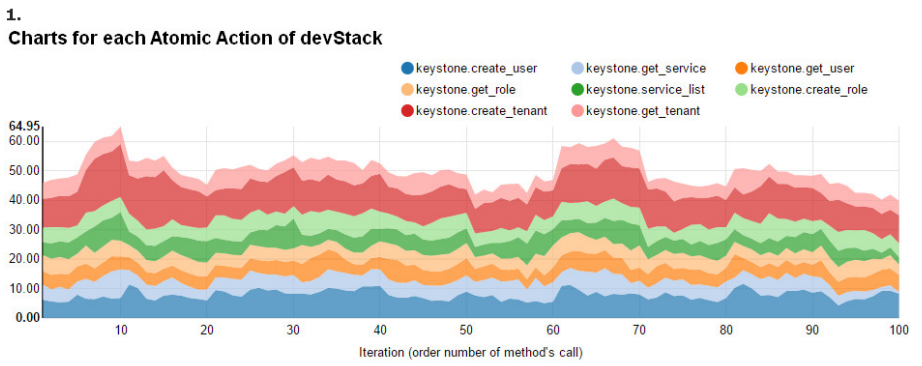

2.

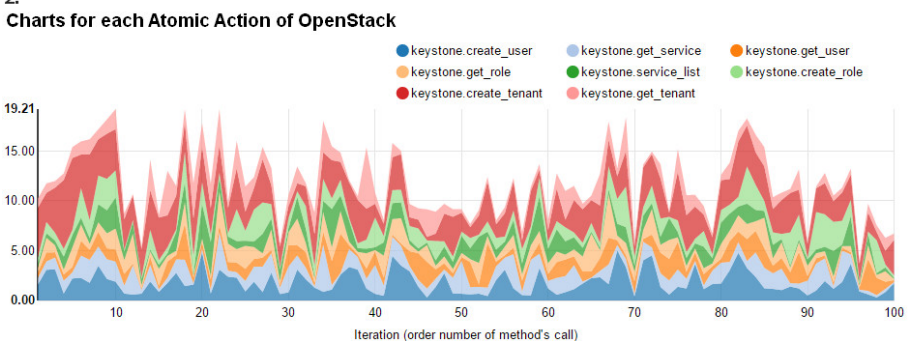

Figure 1. Charts for each Atomic Action in Scenario-1

Scenario 2: In scenario 2, we have run 100 iterations with 2 atomic actions to measure certain glance services correctness and performance. As, shown in Figure 2, x-axis represents number of iterations while the y-axis represents the total duration times to complete the specific iteration in seconds and maximum time is 87.88 ( $\mathrm{sec}$ ) in DevStack case while 44.90 $(\mathrm{sec})$ in OpenStack. As, this scenario also show that OpenStack performance is much better than as compare to DevStack in terms of creating and listing images.

Scenario 3: In scenario 3, we have run 100 iterations with single atomic actions to measure specific nova service correctness. As, shown in Figure 3, x-axis represents number of iterations while the $y$-axis represents the total duration times to complete the specific iteration in seconds and maximum time is $27.90(\mathrm{sec})$ in DevStack case while 2.63 (sec) in OpenStack. As, this scenario also show that OpenStack performance is much better than as compare to DevStack while listing hypervisors.

Analysis of results After observing these scenarios performance, we conclude that although DevStack installer provides quick deployment of OpenStack and it's also much easier to deploy as compare to manual solution but its performance and response time of cloud services is much slower as compare to native OpenStack approach. As, we have tested multiple scenarios from different OpenStack services (e.g. Keystone, Glance, Nova) but response time in each scenario of DevStack is much lower than manual OpenStack. The results shows a noticeable difference in performance for both the testbed platforms which we believe will help the researchers to select

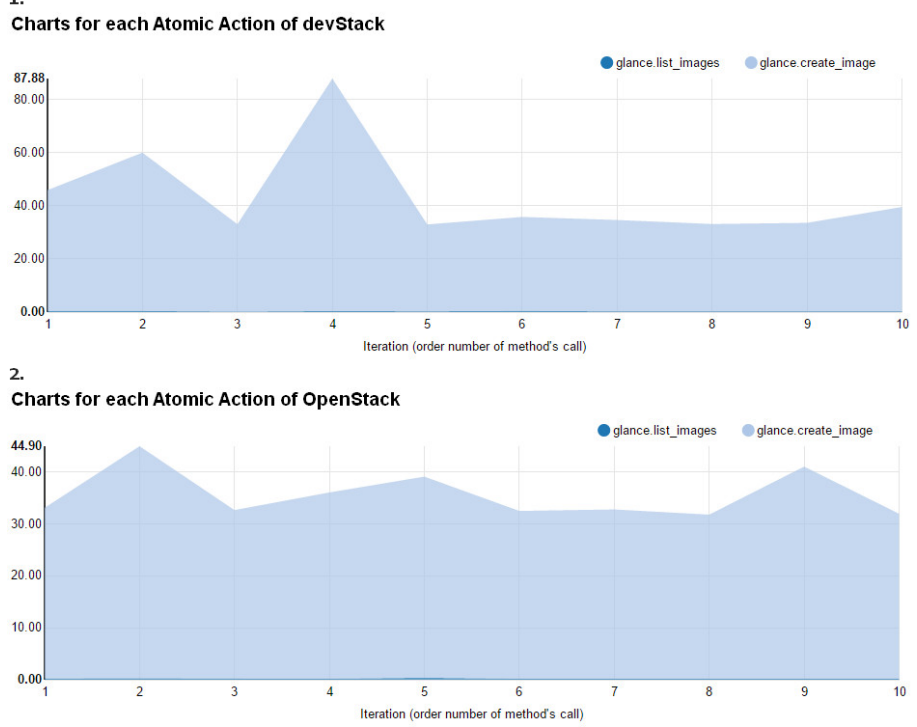

Figure 2. Charts for each Atomic Action in Scenario-2

Charts for each Atomic Action of devStack

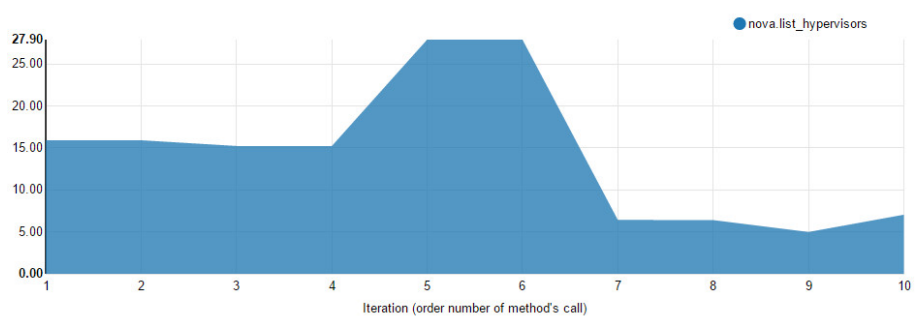

Charts for each Atomic Action of Openstack

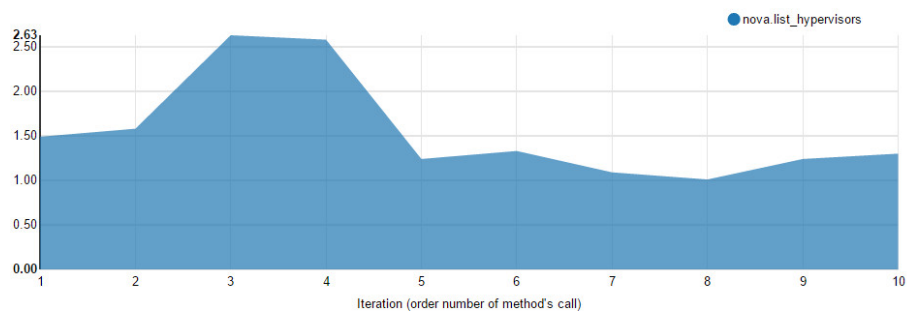

Figure 3. Charts for each Atomic Action in Scenario-3

the most appropriate platform for their testbed deployments.

\section{CONCLUSION AND FUtURE WORK}

Although many cloud platforms are available for both academic and industrial deployments but selecting a most appropriate platform based on user requirements is major challenge. This paper presents a detailed comparison of these platforms while covering different deployment aspects and characteristics such as architecture, networking, supported platforms, hypervisors and languages. Although, each of these platforms has its own strengths and limitations but the presented comparison will help the researchers to select most appropriate platform as per requirement. In this paper, we have also implemented open-source cloud testbed using both 3-tier OpenStack and DevStack platforms and presented performance comparison for both implementations using different 
services scenarios. The results shows that the performance of OpenStack 3-tier implementation is much better as compare to auto deployment method using DevStack. Therefore, the experimentation performed on both the tools using the same hardware and test scenarios may produce different results, which is not acceptable to validate a research hypothese in most of the cases and may raise concerns.

In future work, we will explore the use of light-weight container approaches which is a rapidly growing area of research. We plan to study the performance aspects of using containers and compare it with virtual machines to provide cloud services.

\section{ACKNOWLEDGMENT}

The presented work is being undertaken in the context of the "Self-OrganizatioN towards reduced cost and eNergy per bit for future Emerging radio Technologies" with contract number 734545. The project has received research funding from the H2020-MSCA-RISE-2016 European Framework Program.

\section{REFERENCES}

[1] P. Mell and T. Grance, "The nist definition of cloud computing," 2011.

[2] S. N. R. J. S. Vanishree and P. Karthick, "A prototype for private cloud implementation using open-source platform,"

[3] J. Peng, X. Zhang, Z. Lei, B. Zhang, W. Zhang, and Q. Li, "Comparison of several cloud computing platforms," in Information Science and Engineering (ISISE), 2009 Second International Symposium on, pp. 2327, IEEE, 2009.

[4] S. Wind, "Open source cloud computing management platforms: Introduction, comparison, and recommendations for implementation," in Open Systems (ICOS), 2011 IEEE Conference on, pp. 175-179, IEEE, 2011

[5] M. Armbrust, A. Fox, R. Griffith, A. D. Joseph, R. Katz, A. Konwinski, G. Lee, D. Patterson, A. Rabkin, I. Stoica, et al., "A view of cloud computing," Communications of the ACM, vol. 53, no. 4, pp. 50-58, 2010.

[6] G. Von Laszewski, J. Diaz, F. Wang, and G. C. Fox, "Comparison of multiple cloud frameworks," in Cloud Computing (CLOUD), 2012 IEEE 5th International Conference on, pp. 734-741, IEEE, 2012.

[7] R. Buyya, S. Pandey, and C. Vecchiola, "Cloudbus toolkit for marketoriented cloud computing," in IEEE International Conference on Cloud Computing, pp. 24-44, Springer, 2009.

[8] Q. Zhang, L. Cheng, and R. Boutaba, "Cloud computing: state-of-the-art and research challenges," Journal of internet services and applications, vol. 1, no. 1, pp. 7-18, 2010.

[9] A. Marinos and G. Briscoe, "Community cloud computing," in Cloud Computing, pp. 472-484, Springer, 2009.

[10] C. Vecchiola, S. Pandey, and R. Buyya, "High-performance cloud computing: A view of scientific applications," in Pervasive Systems, Algorithms, and Networks (ISPAN), 2009 10th International Symposium on, pp. 4-16, IEEE, 2009.

[11] D. Durkee, "Why cloud computing will never be free," Queue, vol. 8, no. 4 , p. 20, 2010.

[12] H. BH, L. Suresh, and K. Radhika, "A survey on cloud computing," INTERNATIONAL JOURNAL OF MATHEMATICS, vol. 1, no. 11, 2013

[13] O. Sefraoui, M. Aissaoui, and M. Eleuldj, "Openstack: toward an open-source solution for cloud computing," International Journal of Computer Applications, vol. 55, no. 3, 2012.

[14] R. Prodan and S. Ostermann, "A survey and taxonomy of infrastructure as a service and web hosting cloud providers," in Grid Computing, 2009 10th IEEE/ACM International Conference on, pp. 17-25, IEEE, 2009.

[15] M. Mahjoub, A. Mdhaffar, R. B. Halima, and M. Jmaiel, "A comparative study of the current cloud computing technologies and offers," in Network Cloud Computing and Applications (NCCA), 2011 First International Symposium on, pp. 131-134, IEEE, 2011.
[16] A. Beloglazov, S. F. Piraghaj, M. Alrokayan, and R. Buyya, "Deploying openstack on centos using the kvm hypervisor and glusterfs distributed file system," University of Melbourne, 2012.

[17] G. Raj, C. Kapoor, and D. Singh, "Comparative cloud deployment and service orchestration process using juju charms,"

[18] X. Wen, G. Gu, Q. Li, Y. Gao, and X. Zhang, "Comparison of opensource cloud management platforms: Openstack and opennebula," in Fuzzy Systems and Knowledge Discovery (FSKD), 2012 9th International Conference on, pp. 2457-2461, IEEE, 2012.

[19] “OpenStack." http://www.openstack.org. Accessed: 2017-01-01.

[20] A. Corradi, M. Fanelli, and L. Foschini, "Vm consolidation: A real case based on openstack cloud," Future Generation Computer Systems, vol. 32, pp. 118-127, 2014.

[21] F. Wuhib, R. Stadler, and H. Lindgren, "Dynamic resource allocation with management objectives-implementation for an openstack cloud," in Network and service management (cnsm), 2012 8th international conference and 2012 workshop on systems virtualiztion management (svm), pp. 309-315, IEEE, 2012.

[22] R. Kumar, "Openstack juno release includes features of nfv, big data," Nova, vol. 1, p. 21, 2010.

[23] T. Yanagawa, "Openstack-based next-generation cloud resource management," Fujitsu Sci. Tech. J, vol. 51, no. 2, pp. 62-65, 2015.

[24] M. Bist, M. Wariya, and A. Agarwal, "Comparing delta, open stack and xen cloud platforms: A survey on open source iaas," in Advance Computing Conference (IACC), 2013 IEEE 3rd International, pp. 96100, IEEE, 2013.

[25] T. Cordeiro, D. Damalio, N. Pereira, P. Endo, A. Palhares, G. Gonçalves, D. Sadok, J. Kelner, B. Melander, V. Souza, et al., "Open source cloud computing platforms," in Grid and Cooperative Computing (GCC), 2010 9th International Conference on, pp. 366-371, IEEE, 2010.

[26] Q. Huang, C. Yang, K. Liu, J. Xia, C. Xu, J. Li, Z. Gui, M. Sun, and $\mathrm{Z}$. $\mathrm{Li}$, "Evaluating open-source cloud computing solutions for geosciences," Computers \& Geosciences, vol. 59, pp. 41-52, 2013.

[27] N. G. Bachiega, H. P. Martins, R. Spolon, M. A. Cavenaghi, R. S Lobato, and A. Manacero, "Open source cloud computing: Characteristics and an overview," in Proceedings of the International Conference on Parallel and Distributed Processing Techniques and Applications (PDPTA), p. 237, The Steering Committee of The World Congress in Computer Science, Computer Engineering and Applied Computing (WorldComp), 2013.

[28] H. A. Albaroodi, S. Manickam, and M. F. Aboalmaaly, "The classification and arts of open source cloud computing: A review," Advances in Information Sciences and Service Sciences, vol. 5, no. 16, p. 16, 2013.

[29] F. Meireles and B. Malheiro, "Integrated management of iaas resources," in Euro-Par 2014: Parallel Processing Workshops, pp. 73-84, Springer, 2014.

[30] B. P. Rimal, E. Choi, and I. Lumb, "A taxonomy and survey of cloud computing systems," in 2009 Fifth International Joint Conference on INC, IMS and IDC, pp. 44-51, Ieee, 2009.

[31] I. Nwobodo, "A comparison of cloud computing platforms," 2015.

[32] M. Alam and K. A. Shakil, "Recent developments in cloud based systems: state of art," arXiv preprint arXiv:1501.01323, 2015.

[33] B. Mohammed and M. Kiran, "Analysis of cloud test beds using opensource solutions," in Future Internet of Things and Cloud (FiCloud), 2015 3rd International Conference on, pp. 195-203, IEEE, 2015.

[34] F. Cocozza, G. López, G. Marın, R. Villalón, and F. Arroyo, "Cloud management platform selection: A case study in a university setting," CLOUD COMPUTING 2015, p. 92, 2015.

[35] P. T. Endo, G. E. Gonçalves, J. Kelner, and D. Sadok, "A survey on open-source cloud computing solutions," in Brazilian Symposium on Computer Networks and Distributed Systems, pp. 3-16, 2010.

[36] S. Kumar and R. Goudar, "Cloud computing-research issues, challenges, architecture, platforms and applications: A survey," International Journal of Future Computer and Communication, vol. 1, no. 4, p. 356, 2012.

[37] L. Wang, G. Von Laszewski, A. Younge, X. He, M. Kunze, J. Tao, and C. Fu, "Cloud computing: a perspective study," New Generation Computing, vol. 28, no. 2, pp. 137-146, 2010. 
Table I: Comparison of IaaS Frameworks

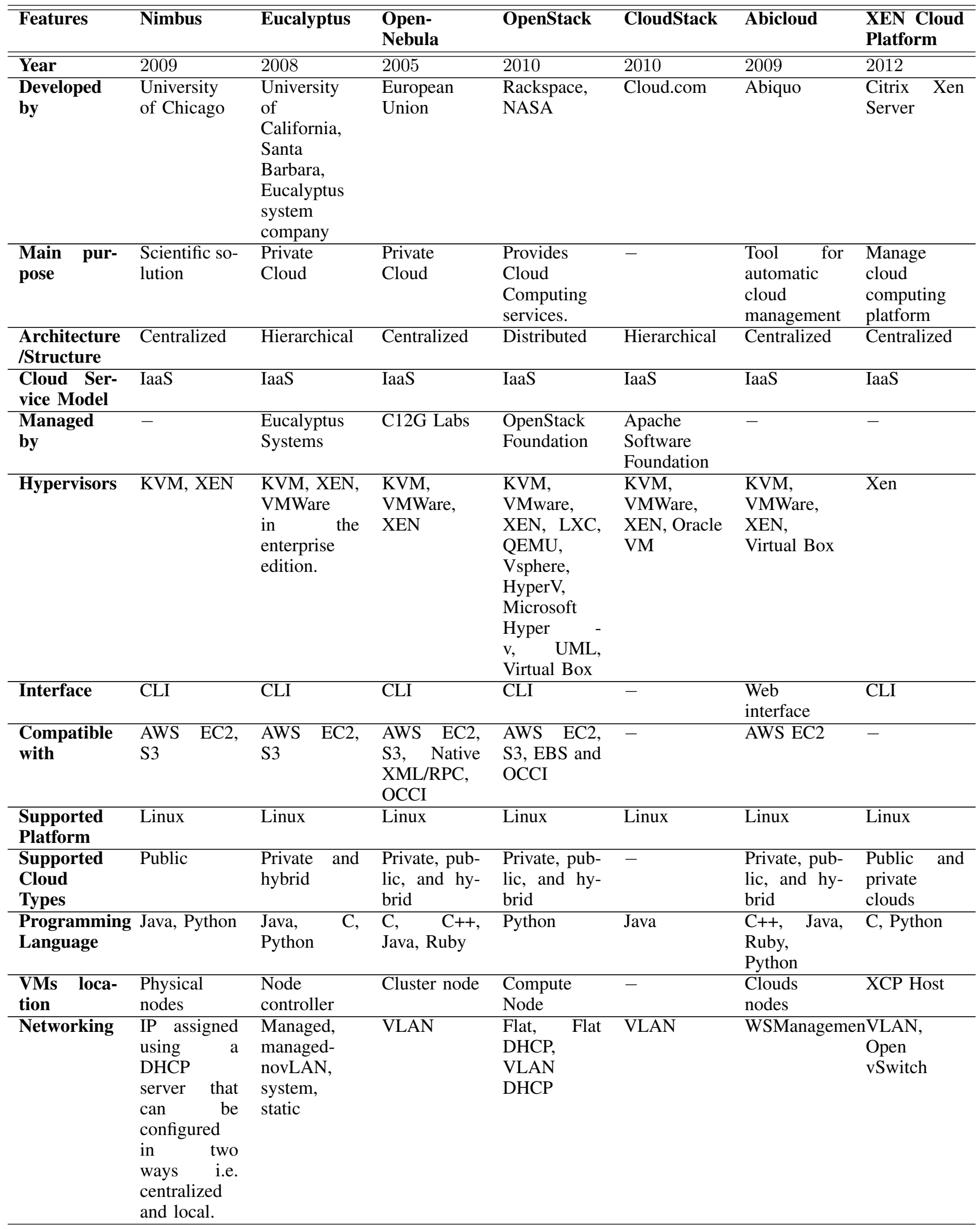




\begin{tabular}{|c|c|c|c|c|c|c|c|}
\hline Storage & - & $\begin{array}{l}\text { Volume and } \\
\text { Object stor- } \\
\text { age }\end{array}$ & $\begin{array}{l}\text { Volume stor- } \\
\text { age }\end{array}$ & $\begin{array}{l}\text { Volume and } \\
\text { Object stor- } \\
\text { age }\end{array}$ & $\begin{array}{l}\text { Volume and } \\
\text { Object stor- } \\
\text { age }\end{array}$ & NFS & $\begin{array}{l}\text { Shared stor- } \\
\text { age }\end{array}$ \\
\hline $\begin{array}{l}\text { DevOps De- } \\
\text { ployment }\end{array}$ & No & $\begin{array}{l}\text { Chef, } \\
\text { Puppet, } \\
\text { Crowbar }\end{array}$ & Chef, Puppet & $\begin{array}{l}\text { Chef, } \\
\text { Puppet, } \\
\text { Crowbar }\end{array}$ & - & - & - \\
\hline Authentication & nX509, Grids & X509 & $\begin{array}{l}\text { X509, } \\
\text { LDAP, } \\
\text { SSH RSA, } \\
\text { key pair, } \\
\text { Password }\end{array}$ & $\begin{array}{l}\text { X509, } \\
\text { LDAP }\end{array}$ & - & $\begin{array}{l}\text { Code } \\
\text { Access Sys- } \\
\text { tem(CAS) }\end{array}$ & - \\
\hline Users & $\begin{array}{l}\text { Scientific } \\
\text { communities }\end{array}$ & Enterprise & Researchers & $\begin{array}{l}\text { Enterprises, } \\
\text { researchers } \\
\text { and } \\
\text { developers. }\end{array}$ & - & Enterprise & - \\
\hline License & $\begin{array}{l}\text { Apache Li- } \\
\text { cense } 2.0\end{array}$ & GPL & $\begin{array}{l}\text { Apache Li- } \\
\text { cense } 2.0\end{array}$ & $\begin{array}{l}\text { Apache Li- } \\
\text { cense } 2.0\end{array}$ & $\begin{array}{l}\text { Apache Li- } \\
\text { cense } 2.0\end{array}$ & GPL & GPL \\
\hline $\begin{array}{l}\text { VM Migra- } \\
\text { tion/Live } \\
\text { Migration }\end{array}$ & No & No & Yes & Yes & Yes & No & Yes \\
\hline $\begin{array}{l}\text { Load } \\
\text { Balancing }\end{array}$ & Yes & - & Yes & Yes & Yes & No & Yes \\
\hline $\begin{array}{l}\text { Fault Toler- } \\
\text { ance }\end{array}$ & Yes & - & Yes & Yes & Yes & No & Yes \\
\hline
\end{tabular}

